\title{
MINI-REVIEW
}

\section{Advanced Diagnostic Aids in Oral Cancer}

\section{KMK Masthan ${ }^{1}$, N Aravindha Babu ${ }^{1}$, Kailash Chandra Dash ${ }^{1}$, M Elumalai ${ }^{2 *}$}

\begin{abstract}
Oral cancers are one of the most common cancers worldwide today. They are usually neglected by the common population when compared to systemic cancers such as the lung cancer, colon cancer etc. However, they also may be extremely fatal if left untreated even at a very initial stage of the lesion. Early detection and treatment gives the best chance for its cure. The five-year survival rate of oral cancer still remains low and delayed diagnosis is suggested to be one of the major reasons. The detection and diagnosis are currently based on clinical examination, histopathological evaluation of the biopsy material and molecular methods. Several diagnostic aids have been developed over the years for early detection of oral cancer. The purpose of this article is to review the advanced available diagnostic adjuncts for the detection of oral cancer.
\end{abstract}

Keywords: Biopsy material, diagnostic adjuncts, histopathology, molecular methods, oral cancer

Asian Pacific J Cancer Prev, 13, 3573-3576

\section{Introduction}

In our oral cavity, oral cancer is a life threatening disease (Burkhardt, 1985). It is a part of group of head and neck cancer which may arise as a primary lesion in any part of the oral cavity or oropharynx by metastasis from a distant site of origin. Oral cancer most commonly involves the tongue, floor of the mouth, buccal mucosa, gingiva and lips. In many Asian countries, especially India, chewing betel, paan and Areca are known to be risk factors for developing oral cancer. Several studies have been done in the past regarding the factors behind the diagnostic delay of Oral Squamous Cell Carcinoma (OSCC) but early detection of it still remains disappointingly constant over recent decades. OSCC can be a small problem in numerical terms, but it is considered as a highly lethal disease in world population (Binnie and Rankin, 1984). Lack of awareness in the public of the various signs, symptoms and risk factors for oral cancer are all believed to be responsible for the diagnostic delay in a long venture (Stefano, 2009). They are often difficult to diagnose by routine clinical examination. Diagnosis of these diseases is mostly based on the microscopic study of cells and tissues (Richard et al., 2002). Maryland, a District of Columbia has seventh highest overall mortality rate for oral cancer in the state, due to lack of information regarding educational materials and interventions for the public to promote oral cancer (Horowitz et al., 2002). Most of the oral cancers are OSCC. In past three decades, the five-year survival rate has improved but still remains in the range of 53\% to $60 \%$. Most OSCC is not diagnosed until an advanced stage, which has been one of the major reasons for minimally improved survival rate over the years (Jemal et al., 2009; Yi-Shing, 2011). Historically, the screening of patients with signs and symptoms of oral cancer and various precancerous lesions has usually been relied upon the conventional pattern, that is, oral examination (Lingen et al., 2008). Application of immunohistochemistry has proved to be a useful tool, laboratory diagnosis in oral cancer by the use of antigens and antibodies (Pettigrew, 1989). An early detection of these cancers helps in better and faster treatment for improving the prognosis to some extent and the available advanced diagnostic adjuncts aid as a helpful tool for the early diagnosis of oral cancer to the medical practitioners in treating patients suffering from it.

Table 1. Advanced Diagnostic Aids for Oral Cancer

Clinical Methods

Vital staining - Toluidine Blue/Lugol's Iodine

Vizilite

Brush Cytology

Visualization Adjuncts Tissue Auto fluorescence

VELscope

In Vivo Confocal Microscopy

Saliva-based oral cancer diagnostics

Molecular Methods

DNA Ploidy \& Quantification of nuclear DNA content Tumor Markers \& Bio Markers

PCR-Based diagnostic aids

Photo diagnosis

Auto fluorescence Spectroscopy

Fluorescence Photography 


\section{Vital Tissue Staining - Toludine Blue Staining \& Lugol's Iodine}

Oral carcinoma in situ and early invasive oral carcinoma shows affinity for toluidine blue dye. Lugol's iodine and toluidine blue have been used together in the detection of early carcinomas and other oral lesions.

Toluidine blue is an acidophilic meta chromatic dye which selectively stains acidic tissue components, thus staining DNA and RNA. As it binds to nucleic acids (DNA or RNA), it helps in better visualization of high risk areas especially with rapid cell proliferation of OSCC and premalignant lesions (Pegah et al., 2012). It stains mitochondrial DNA, cells with greater than normal DNA content or altered DNA seen in dysplastic and malignant cells. Lugol's solution is used for delineation of the malignant change which produces a brown black stain when the iodine reacts with the glycogen content. The use of toluidine blue and Lugol's iodine serves as a useful adjunct in the diagnosis of patients who are at risk and for selecting the site for biopsy with wide field cancers prior to treatment (Sujata and Ajit, 2006).

\section{Vizilite}

Vizilite is a non toxic chemiluminescent light. Today, vizilite Plus examination, in combination with the regular visual examinations, provides a comprehensive oral screening procedure for those patients who are at increased risk for oral cancer. Vizilite Plus technology helps in identifying soft tissue abnormalities which is shined inside the mouth. This shows glowing of abnormal tissue different from that of normal tissue thus making it more visible. The technique is painless and fast and can help in saving life. However, it cannot necessarily tell if they are potentially cancerous or not (Sujata and Ajit, 2006). To improve early detection of oral cancer, the use of a dilute acetic acid rinse and observation under a chemiluminescent light such as ViziLite is usually recommended (Oh and Laskin, 2007).

\section{Brush Cytology}

Brush cytology (Oral CDX), developed in 1999 and has become popular in dental practice today. In the past decades, adjunctive technique has facilitates the early detection of oral premalignant and malignant lesions (OPML). In that context, Oral CDx is useful in the assessment of dysplastic changes in various suspected lesions especially in oral cancer (Patton et al., 2008). As majority of oral cancers are squamous cell carcinomas, Cytological study of oral cells is a relatively inexpensive, simple, noninvasive and also risk-free technique which is well accepted by the patient and medical practitioner today (Smaroula et al., 2009). The oral cells can be obtained by the use of a cytobrush. With brush cytology, sensitivity for detecting oral epithelial dysplasia or Oral squamous cell carcinoma is high (Yi-Shing and John , 2011). But, the technique has attracted lots of controversies and more incidences of false negative results with this technique (Sujata and Ajit, 2006) has been encountered.

\section{VEL scope}

VEL scope is a hand-held device which was approved by Federation Dentaire Association for direct visualization of autofluorescence in the oral cavity. Only recently it was introduced in the market as a diagnostic adjunct for oral cancer detection. The VEL scope Vx is one of the most powerful tools available today for assisting in oral abnormalities especially oral cancer. The distinctive blue-spectrum light causes the soft tissues of the mouth to naturally fluoresce. The use of VEL scope Vx is a safe and simple technique and the entire examination can be done in about two minutes. However, it is a relatively new device and so far only a limited number of studies have been done on its effectiveness as a diagnostic adjunct for oral cancer (Yi-Shing and John, 2011).

\section{In Vivo Confocal Microscopy}

Confocal microscopy is an imaging technique for various researches in cell biology with an advantage of optical sectioning and high resolution imaging. In vivo confocal images from the oral cavity show the characteristic features such as nuclear irregularity which is used to differentiate OSCC from normal oral mucosa. However, further optimization of the instrument is still needed to rate it a promising non-invasive tool for the early detection of oral cancer (Yi-Shing and John, 2011).

\section{Saliva-based oral cancer diagnostics}

The concept of saliva to diagnose OSCC is a latest concept. Oral fluid or the saliva is a noninvasive, accessible and highly efficient diagnostic medium today. The utility of salivary transcriptome diagnostics are helpful in the detection of oral cancer (Li et al., 2004). Promoter hypermethylation patterns of TSG p16, O6methyl guanine-DNA-methyltransferase, and death associated protein kinase are identified in the saliva of head and neck cancer patients and high salivary counts of Capnocytophaga gingivalis, Prevotella melaninogenica and Streptococcus mitis is found in patients with OSCC. However, it is still difficult to support the suggestion that this could be a reliable diagnostic indicator (Crispian et al., 2008). Today, saliva testing for genetic patterns which are linked with oral cancer is gaining interest of research but still it has not been incorporated as a commercial product, but researchers are hopeful that this technology will be readily available in the market very soon (Tricia and Suzie, 2008). One of the factor behind oral cancer's such high mortality is failure in detecting it at an early stages. The use of saliva for the detection of oral cancer has proved to be a historical goal that has to be reached to the population in the future for better and faster management of OSCC (Wong, 2006).

\section{DNA Ploidy \& Quantification of nuclear DNA content}

DNA ploidy is the measurement of nuclear DNA content that provide a measurement of gross genetic 
damage. If the chromosomes are not uniformly distributed to the daughter cells during mitosis or if some parts of chromosomes become detached, the chromosomal segregation becomes unbalanced and aneuploidy is seen which is commonly observed in many cancers. DNA image cytometry shows high sensitivity and serves as a non-invasive method for cancer (Crispian et al., 2008). Pre-malignant lesions such as oral leukoplakias, the nuclear DNA distribution patterns can be analyzed by flow-cytometry, showing different rates of dysplasia, however the quantity of specimens should be more for the examination ( Grässel-Pietrusky et al., 1982 ). Even cytology with DNA-cytometry has emerged as a highly sensitive and non-invasive method for the early diagnosis of oral epithelial neoplasia and hence in oral cancer (Maraki et al., 2004).

\section{Tumor Markers \& Bio Markers}

Tumor markers may be present in blood circulation, body cavity fluids, cell membranes and cell cytoplasm when released by cancer cells or produced by the host in response to cancerous substances. They are used in identification of a cancerous growth (Sujata Satoskar and Ajit Dinakar, 2006). Tumour Suppressor Genes, oncogenes, cell proliferation markers, angiogenic markers and cell adhesion molecules are some of the potential tools which help in prediction for the prognosis of patients with OSCC. According to a study, use of cytokeratin markers are also used in detecting OSCC by the help of analyzing the altered keratin expression in the oral site especially the buccal mucosa (Vaidya et al., 1989).

\section{PCR-Based diagnostic aids}

The polymerase chain reaction (PCR) is a scientific technique in molecular biology which can be used in the diagnosis and study of infectious diseases and malignancies associated with micro organisms. PCR helps in the study of cancer and provide clearer understanding of the pathogenesis of neoplasia. PCR can be used to detect mutations in cancer-associated oncogenes (e.g., K-ras, Nras), tumor suppressor genes (e.g., p53, p16) etc. and aids as an important detection tool (Richard et al., 2001). PCR technique has increased the range and sensitivity of diagnostic procedures but still with a major drawback, as contamination and amplification artefacts may give rise to difficulties in the interpretation of the desired results (O'Leary et al., 1997). With the introduction of polymerase chain reaction (PCR), reverse transcriptase PCR (RT-PCR) and other molecular techniques, the diagnosis and prognosis of other lesions such as chronic myelogenous leukemia has also been useful (Glassman, 1998).

\section{Auto fluorescence Spectroscopy}

Auto fluorescence spectroscopy has emerged as a promising tool for oral cancer detection. The system consists of a small optical fiber which produces various excitation wavelengths and a spectrograph which receives and records on a computer and analyzes it with the help of software, the spectra of reflected fluorescence from the tissue. However, the technique is controversial and often found with unclear results. Overall, it seems to be very accurate for distinguishing lesions especially malignant tumors from healthy oral mucosa, with a high sensitivity and specificity (Stefano Fedele, 2009). It is a non-invasive aid in the detection of various alterations in the structural and chemical compositions of cells indicating the presence of a diseased tissue. It can be useful in guiding the clinician in identifying the optimal location for biopsy (Sujata Satoskar and Ajit Dinakar, 2006). According to a study, on using violet excitation light, camera-based autofluorescence photodetection technique has presented as a highly promising tool for the diagnosis of oral malignancies (Betz et al., 1999)

\section{Fluorescence Photography}

Fluorescence photography is non-invasive, rapid, simple and reproducible method in detection of oral cancer. Fluorescence positivity can show enlargement of carcinomas and progression of the disease. The system is usually used in the diagnosis of squamous cell carcinoma. However, biopsies are still necessary (Sujata and Ajit, 2006). According to a study, fluorescence photography has shown as a useful tool for the diagnosis of oral cancer, especially in patients with squamous cell carcinoma (Onizawa et al., 1996).

\section{PET scan}

Our oral cavity is one of the cancer sites in the head and neck which is accompanied by a high incidence of regional metastasis. Due to cervical lymph node metastasis, there is significantly reduction in the survival of the patient and which is an important prognostic factor for scientific debate today. Positron emission tomography (PET) with fluorodeoxyglucose (FDG) is increasingly day by day as a useful tool in preoperative staging of cancer patients (Bart et al., 2006). According to recent studies and research, FDG-PET scanning shows good accuracy and predictive value in determining lymph node status and thus helping in screening and early diagnosis of oral cancer in affected patients. Standardized uptake value (SUV) of the tumor mass helps in prognosis for overall survival of the affected patient (Gregory et al., 2010).

\section{Conclusion}

To conclude this review, regardless of all the type of oral cancer, it is still unknown what really causes its development. Recent studies show that usage of tobacco may be the leading cause of oral cavity and oropharyngeal cancer development and it is believed that smokers are more likely to develop oral cancer than nonsmokers. Carcinogens, which are present in high concentration in tobacco and its products and are also the leading cause of cancer in lungs, esophagus and several other organs. Screening and early detection of oral cancer using various diagnostic aids mentioned herewith decrease the risk of 


\section{K M K Masthan et al}

morbidity and mortality associated with oral cancer. There has been a dramatic increase in the development of many potential oral cancer screening techniques in last few years and still many researchers are on the look for any better and faster aids of diagnosing these life threatening cancers.

\section{References}

Bart Wensing M, Wouter Vogel V, Henri Marres A M, et al (2006). FDG-PET in the clinically negative neck in oral squamous cell carcinoma. Laryngoscope, 116, 809-13

Betz CS, Mehlmann M, Rick K, et al (1999). Autofluorescence imaging and spectroscopy of normal and malignant mucosa in patients with head and neck cancer. Lasers Surg Med, 25, 323-34.

Binnie WH, Rankin KV (1984). Epidemiological and diagnostic aspects of oral squamous cell carcinoma. J Oral Pathol, 13, 333-41.

Burkhardt. A, (1985). Advanced methods in the evaluation of premalignant lesions and carcinomas of the oral mucosa. review article. J Oral pathology, 14, 751-78.

Crispian Scully, José Bagan V, Colin Hopper, Joel Epstein B (2008). Oral cancer: Current and future diagnostic techniques. Am J Dentistry, 21, 199-209.

Glassman AB (1998). Cytogenetics, in situ hybridization and molecular approaches in the diagnosis of cancer. Ann Clin Lab Sci, 28, 324-30.

Grässel-Pietrusky R, Deinlein E, Hornstein OP (1982). DNAploidy rates in oral leukoplakias determined by flowcytometry. J Oral Pathol, 11, 434-8

Gregory Kubicek J, Collin Champ, Shannon Fogh, et al (2010). FDG-PET staging and importance of lymph node SUV in head and neck cancer. Head \& Neck Oncology, 2, 1-7

Horowitz AM, Canto MT, Child WL (2002). Maryland adults perspectives on oral cancer prevention and early detection. J Am Dent Assoc, 133, 1058-63.

Jemal A, Siegel R, Ward E, Hao Y, Xu J, Thun MJ. (2009). Cancer statistics, 2009. CA Cancer J Clin, 59, 225-49.

Li Y, St John MA, Zhou X et al. (2004). Salivary transcriptome diagnostics for oral cancer detection. Clin Cancer Res, $\mathbf{1 0}$, 8442-50.

Lingen MW, Kalmar JR, Karrison T, Speight PM (2008). Critical evaluation of diagnostic aids for the detection of oral cancer. Oral Oncol, 44, 10-22.

Maraki D, Becker J, Boecking A. (2004). Cytologic and DNAcytometric very early diagnosis of oral cancer. J Oral Pathol Med, 33, 398-404.

Oh ES, Laskin DM. (2007). Efficacy of the ViziLite system in the identification of oral lesions. J Oral Maxillofac Surg, 65, 424-6

O’Leary J J, Engels K, Dada M. A(1997). The polymerase chain reaction in pathology. J Clin Pathol, 50, 805-10

Onizawa K, Saginoya H, Furuya Y, Yoshida H. (1996). Fluorescence photography as a diagnostic method for oral cancer. Cancer Lett, 108, 61-6.

Patton LL, Epstein JB, Kerr AR. (2008). Adjunctive techniques for oral cancer examination and lesion diagnosis: a systematic review of the literature. J Am Dent Assoc, 139, 896-905.

Pegah Mosannen Mozafari, Zahra Delavarian, Nooshin Mohtasham (2012). Diagnostic Aids in Oral Cancer Screening. Oral cancer, 189-208

Pettigrew NM (1989). Techniques in immunocytochemistry. Application to diagnostic pathology. Arch Pathol Lab Med, 113, 641-4.

Richard Jordan CK., Troy Daniels E, John Greenspan S, Joseph
Regezi A (2002). Advanced diagnostic methods in oral and maxillofacial pathology. Part II: Immunohistochemical and immunofluorescent methods. Oral Surgery Oral Med Oral Pathology, 93, 56-74.

Richard Jordan CK., Troy Daniels E, John Greenspan S, Joseph Regezi A (2001). Advanced diagnostic methods in oral and maxillofacial pathology. Part I: Molecular methods. Oral Surgery Oral Med Oral Pathology, 92, 650-69.

Smaroula Divani, Maria Exarhou, Leonidas-Nectarios Theodorou, Dimitrios Georgantzis, Haralambos Skoulakis (2009). Advantages and difficulties of brush cytology in the identification of oral cancer. Arch Oncol, 17, 11.

Stefano Fedele (2009). Diagnostic aids in the screening of oral cancer. Head \& Neck Oncology, 1, 1-6.

Sujata Satoskar, Ajit Dinakar (2006). Diagnostic aids in early oral cancer detection- a review. J Indian Academy of Oral Med \& Radiol, 18, 82-9.

Tricia Osuna, Suzie Hopkins (2008). Oral Cancer Diagnostic Technologies, CDHA J, 24, 12-18.

Vaidya MM, Borges AM, Pradhan SA, Rajpal RM, Bhisey AN (1989). Altered keratin expression in buccal mucosal squamous cell carcinoma. J Oral Pathol Med, 18, 282-6.

Wong DT (2006). Salivary diagnostics for oral cancer. J Calif Dent Assoc, 34, 303-8.

Yi-Shing Lisa Cheng, John Wright (2011). Advances in Diagnostic Adjuncts for Oral Squamous Cell Carcinoma. The Open Pathology J, 5, 3-7. 